\title{
Early qualitative risk assessment of the emerging zoonotic potential of animal diseases
}

\author{
Stephen Palmer, David Brown, Dilys Morgan
}

Most new human infections are of animal origin, but there is rarely sufficient evidence to make a risk assessment of the zoonotic potential of emerging animal diseases. An algorithm for early qualitative public health risk assessment has been developed to guide risk management

Department of Epidemiology, Statistics and Public Health, Cardiff University, Cardiff CF4 4XN Stephen Palmer Mansel Talbot professor of epidemiology and public health

Health Protection Agency Centre for Infections, London NW9 5HT

David Brown consultant virologist Dilys Morgan consultant epidemiologist Correspondence to: S Palmer palmersr@ cardiff.ac.uk
Most newly emerging human infections of global importance are of animal origin, ${ }^{1 \mathrm{w} 1}$ but early accurate predictions of zoonotic risk of emerging animal diseases are difficult, as shown by the epidemic of bovine spongiform encephalopathy and variant Creutzfeldt-Jakob disease in the United Kingdom. ${ }^{\text {w2 }}$ Miscalculation, delays, or poor risk communication can result in failure to protect the public's health and undermine public trust, ${ }^{2}$ but overreaction can waste resources and even harm the economy of countries, as in the case of suspected plague in India. ${ }^{3}$ The public are increasingly anxious to understand the basis on which a government's decisions on risk management are taken, ${ }^{\mathrm{w} 3}$ but scientists and government may be reluctant to engage with the public at an early stage because of the fear of provoking a public scare. However, since the BSE epidemic, it has been accepted that the criteria used and the evidence considered in risk assessments should be open and explicit. ${ }^{4} \mathrm{w}^{4} \mathrm{w} 5$

In this article we describe and illustrate such an approach, one that explicitly distinguishes evidence of lack of zoonotic potential from lack of evidence. The algorithm, endorsed by the UK government's Zoonoses Group $^{w 6}$ and by the National Expert Panel on New and Emerging Infection, ${ }^{w 7}$ is being used by the Human and Animal Infections and Risk Surveillance Group of the Health Protection Agency. ${ }^{5}$

\section{Methods}

The algorithm (figure) is used to categorise the evidence of zoonotic potential into four levels (box 1) by considering three key stages in the transmission of zoonoses:

The risk of cross species transmission-Seek to identify criteria that might exclude the possibility of human infection if exposure occurred (box 2). If these criteria do not support human infection, or there is no equivalent disease process in humans, it is defensible to take no further action. If human infection is biologically plausible then the availability and use of a serological or molecular test for exposed humans should be considered.

Exposure of humans to infected animals and secondary sources-The full range of possible exposures needs to be addressed systematically, including occupational, recreational, domestic, food and waterborne, and travel. Negative serosurveys reduce concern about the zoonotic potential.

Human infection and subsequent human to human transmission-The absence of reported cases must be assessed in relation both to opportunities for exposure and the sensitivity of surveillance systems to detect human cases. At each point of assessment, the quality and completeness of available data must be considered.

\section{Box 1: Levels of confidence of risk of zoonotic transmission of animal diseases}

Level 0:Not zoonotic-Evidence of lack of zoonotic potential. Good grounds for not taking further action Level 1: Potential zoonosis-Possibility of human pathogenicity not excluded. Work needed on biomarkers of infection and pathways of exposure Level 2:Potential zoonosis-Serological evidence of infection, or human exposure has occurred but surveillance not sufficiently reliable. Enhanced surveillance needed

Level 3: Confirmed zoonosis-Human cases have been reported, but evidence against person to person spread. Enhanced surveillance needed. Control exposure of humans to animals and environmental sources

Level 4: Confirmed zoonosis-Human cases have occurred, with subsequent person to person spread not excluded. Control of direct or indirect person to person spread needed

\section{Worked examples}

The algorithm was applied to five emerging animal diseases of concern to public health authorities. A possible link between porcine dermatitis and nephropathy syndrome (PDNS) and Henoch-Schönlein purpuraw8 was raised by medical practitioners in East Anglia and was referred to the Public Health Laboratory Service Advisory Committee on Zoonoses by the Department of Health. Clostridium difficile in pigs was referred to the advisory committee by the director of the Public Health Laboratory Service because of recent reports from the United States that piglet neonatal enteritis due to $C$ difficile had become epidemic there, ${ }^{\text {w9 }}$ and that typing studies had suggested that pig isolates had also been documented in human cases. Porcine hepatitis $\mathrm{E},{ }^{\mathrm{w} 10}$ bovine norovirus, ${ }^{\mathrm{w} 11}$ and Borna disease viruses ${ }^{\mathrm{w} 12}$ were identified by the advisory committee itself as priorities for assessment

\section{Porcine hepatitis E}

Pathogen-Hepatitis E virus. ${ }^{6}$

Agent characteristics-Unclassified and is recognised in pigs, rats, and humans with no important virological differences in the agents.

Disease characteristics-Usually a self limiting illness, but fulminant hepatitis is recognised in high risk groups such as pregnant women. ${ }^{\mathrm{w} 10}$ No disease is identified in pigs. Hepatitis $\mathrm{E}$ is a recognised human infection in the UK, mainly associated with travel abroad.

Extra references w1-w36 appear on bmi.com 


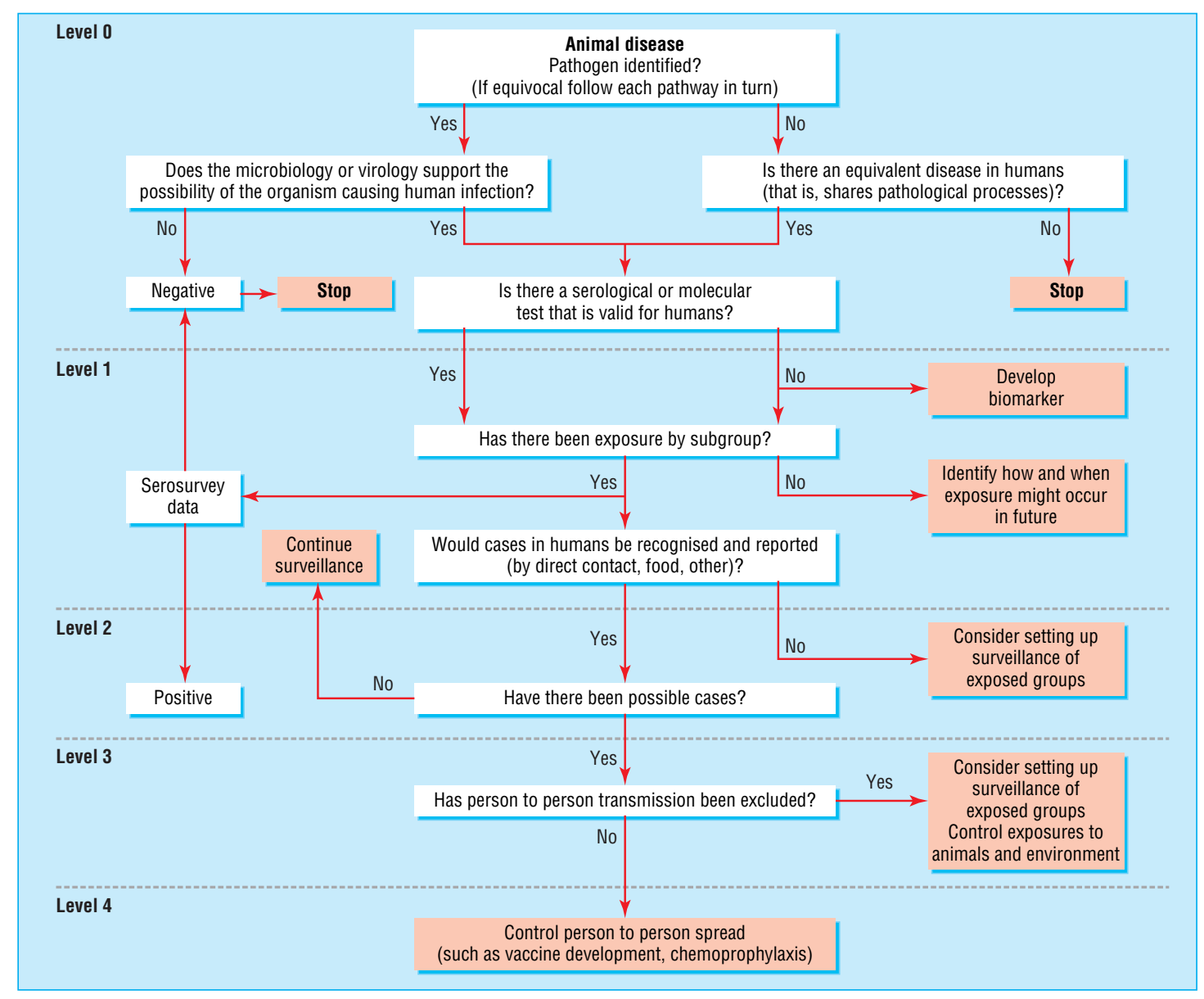

Template for qualitative risk assessment of zoonotic potential of animal diseases

Biomarkers in humans-Virological diagnosis is possible by serology and virus characterisation by the polymerase chain reaction and sequencing. ${ }^{\mathrm{w} 13}$

Exposure-Infection is common in UK pigs, ${ }^{\mathrm{w} 14}$ and direct occupational human exposure is likely. Occupation exposure studies have not been carried out to our knowledge in the UK. Virus was considered likely to contaminate food and the environment.

Diagnosis or recognition-Serological diagnosis is available, but testing is not routine for hepatitis cases in the UK without a history of foreign travel; therefore, sporadic cases may well be missed.

\section{Box 2: Some virological criteria to consider to} assess zoonotic risk of viral infections

\section{Viral factors}

- Virus family, genus, and species

- Evidence of interspecies transmission in virus family

- Any related human viruses

- Phylogenetic relationship with human strains

- Genetic stability or diversity RNA or DNA

Segmented genome

- Culturable in human cells

Virus host factors

- Virus specific host receptors

- Poikilothermic $v$ homoeothermic body temperature
Putative cases-Human cases of hepatitis E associated with pigs have been reported since the appraisal was conducted. ${ }^{7}$ There have also been few human cases in the UK with no foreign travel history from whom hepatitis $\mathrm{E}$ with a sequence very close to the European pig strain have been characterised. In other countries serosurveys show higher prevalence of infection with hepatitis $\mathrm{E}$ virus in veterinarians and pig farmers than controls. ${ }^{\mathrm{w} 15}$

Person to person transmission-No cases documented yet to our knowledge.

Conclusion and recommendation-Level 2 zoonosis. Porcine hepatitis E might cause human infections, but sporadic cases in the UK may be missed since the epidemiology of human infections is lacking. We cannot therefore be confident that the absence of reported cases indicates absent or low risk. Enhanced surveillance of cases of non-A, non-B, non-C hepatitis should be instituted, and occupational exposure studies should be carried out.

\section{Porcine circovirus}

Pathogen-Circovirus infection is believed to be associated with post-weaning multi-systemic wasting syndrome (PMWS) and PDNS disease in pigs, but its aetiological agent is still debated. ${ }^{8}$

Agent characteristics-Circovirus is similar to but distinct from human transfusion transmitted (TT) virus. ${ }^{\mathrm{w} 16}$ There are insufficient direct studies looking for circovirus in humans to exclude the possibility of human infection. 
Disease characteristics-Suggested similarities between the pathology of Henoch-Schönlein purpura in humans and PDNS led to the review. ${ }^{\mathrm{w} 14}$

Biomarkers in humans-Virological diagnosis by serology and virus characterisation by the polymerase chain reaction and sequencing is possible. ${ }^{\mathrm{w} 16}$

Exposure-The disease is widespread in pigs, ${ }^{\mathrm{w} 14}$ and the agent could contaminate meat and the environment.

Diagnosis or recognition-There is no surveillance system for Henoch-Schönlein purpura, and human cases occurring in conjunction with infected pigs could easily be missed. However, a US serosurvey of 50 veterinarians exposed to PMWS found no seropositive cases. ${ }^{9}$ Surveys of blood donors have also proved negative. ${ }^{\mathrm{w} 16}$

Putative cases-Time trends and geographical patterns of human Henoch-Schönlein purpura across England and Wales have been compared with the evolution of the PMWS epidemic, and there is no evidence of a link between the two diseases. ${ }^{\text {w14 }}$

Conclusion and recommendation-Level 0 zoonosis. Though theoretically plausible, infection has not been shown in humans exposed to infected pigs. The epidemiological pattern of Henoch-Schönlein purpura is not related to the epidemiology of PMWS in the UK. To date, there is some evidence that porcine circovirus is not a significant zoonotic risk to occupationally exposed groups. Further serological studies in exposed groups in the UK would strengthen this conclusion.

\section{Bovine norovirus}

Pathogen-Norovirus (previously known Norwalk-like or small round structured virus). ${ }^{\mathrm{w17}}$

Agent characteristics-Noroviruses cause infection and illness in cattle, pigs, and humans. Virological studies suggest recombination events are common. ${ }^{\mathrm{w} 18}$ Interspecies transmission is established for viruses belonging to the caliciviridae family. ${ }^{\mathrm{w} 19}$

Disease characteristics-Gastroenteritis in humans and cattle.

Biomarkers in humans-The polymerase chain reaction and sequencing are widely used for diagnosis and would identify bovine strains in human infections. Serology is available by means of recombinant capsids. $^{\text {w19 }}$

Exposure-Bovine infection is widespread, and human occupational exposure is likely. In theory milk might become contaminated, although pasteurisation should destroy the virus. Shellfish contamination was also considered possible through slurry.

Diagnosis or recognition-Viral gastroenteritis in humans is so common that sporadic cases from bovine contact are unlikely to be recognised epidemiologically. ${ }^{\text {w20 }}$

Putative cases-Several thousand strains of norovirus from human infections have been sequenced over the past 10 years, and no porcine or bovine norovirus strains have been detected among these strains infecting humans. ${ }^{10}{ }^{11}$

Conclusion and recommendation-Level 0 zoonosis. There is good evidence that bovine norovirus is not a significant zoonosis, even though agent characteristics provide grounds for concern about zoonotic potential. No specific action is recommended.

\section{Borna disease virus}

Pathogen-Borna disease virus causes a neurological disease of horses and other domesticated animals. ${ }^{12}$

Agent characteristics-Wide host range, high sequence homology of virus isolates has been reported. ${ }^{12}$

Disease characteristics-Causes behavioural abnormalities in experimentally infected primates. ${ }^{12}$

Biomarkers in humans-Serological and molecular tests have been developed for human samples, but their specificity is still debatable. ${ }^{13}$

Exposure-Human exposure to infected animals is widespread in central Europe.

Diagnosis or recognition-Borna disease virus has been proposed as the cause of a range of common chronic diseases-including depression, schizophrenia, and chronic fatigue syndrome-but it is most unlikely that occasional cases of mental illness caused by the virus would be identified.

Putative cases-Cases have been proposed, but diagnostic and serological studies are controversial. ${ }^{13}$ There is some evidence of increased prevalence of antibodies to the virus in certain patient groups (such as patients with schizophrenia or neurological disease). ${ }^{14}$

Person to person spread-Higher seroprevalence has been reported in relatives of schizophrenic patients and mental health workers. Family clusters of Borna disease have been reported in Germany. ${ }^{13}$

Conclusion and recommendation-Level 2 zoonosis. The weakness in the evidence of the zoonotic potential of Borna disease virus is because of the uncertain specificity of human serological tests, and therefore the interpretation of serosurveys in humans. Better serological and molecular tests should be developed and applied to exposed groups.

\section{Clostridium difficile}

Pathogen-Clostridium difficile. ${ }^{15}$

Agent characteristics-Widespread in animal hosts and the environment, with many subtypes shared between animals and humans. ${ }^{\mathrm{w} 21}$ The $\mathrm{a}$ and $\mathrm{b}$ toxins have been shown to cause disease in humans. ${ }^{15}$

Disease characteristics-Pseudomembranous colitis is seen mainly in people aged $>65$ years who have recently had antimicrobial treatment. ${ }^{\text {w22 }}$

Biomarkers in humans-Reliable tests to detect both $\mathrm{a}$ and $\mathrm{b}$ toxins in faeces are available commercially. ${ }^{15} \mathrm{C}$ difficile may also be cultured from faeces of patients with diarrhoea caused by toxins in the gut. ${ }^{15}$

Exposure-Occupational human exposure to infected or colonised animals was thought probable.

Diagnosis or recognition-Strains of $C$ difficile causing animal disease have also been identified in human cases. $^{\text {w21 }}$

Putative cases-No human cases linked to specific animal infection have yet been reported.

Person to person spread-Cross infection can occur in hospital wards. ${ }^{\text {w22 }}$

Conclusion and recommendation-Level 2 zoonosis. Human exposure from animal cases is likely, but, since human infection usually only occurs in debilitated patients who have recently taken antibiotics, zoonotic cases are unlikely. However, current surveillance is not sensitive enough to detect such sporadic occurrences. No additional action is recommended at this time. 


\section{Discussion}

We sought explicit criteria to judge the possibility of animal infections causing human diseases. However, even in the special case of xenotransplantation there does not seem to be an agreed approach, beyond "sound professional judgment" of panels of experts representing all scientific fields. ${ }^{16}{ }^{\mathrm{w} 23}$ Special attention has been devoted in the literature to porcine endogenous retroviruses, ${ }^{\text {w24 }}{ }^{\text {w25 }}$ where nucleic acid sequence homology, cellular receptors, and the potential for protein and gene expression suggest multiple possible sites for infection in humans. ${ }^{\text {w26 }}$ However, the real test of zoonotic potential, the occurrence of infection in exposed people, has not been found, although study numbers are small. ${ }^{17}$

Only rarely will the biological evidence available for risk assessment be so detailed as for porcine endogenous retroviruses. A more common scenario is illustrated by Audelo-del-Valle et $\mathrm{al},{ }^{18}$ who argue that Taura syndrome virus of penaeid shrimps is a member of the "picornavirus superfamily," which includes human pathogens, and that it could "potentially represent a public health threat." However, the questions for public health are how much weight should be given to this concern and what resources should be given to assessing the risk. The authors reported infection of cultured human and monkey cell lines with Taura syndrome virus, ${ }^{18}$ but others have failed to replicate the findings. ${ }^{19}$

At current levels of knowledge, only rarely could the possibility of human infection be excluded on microbiological or virological grounds, and as virological knowledge increases even well established human infections may be recognised as zoonoses, as in the case of rotavirus. ${ }^{20}$ w27 Absence of growth in human cells in culture is not a reliable criterion, since many human viruses do not grow in commonly used cell cultures (such as norovirus). ${ }^{28}$ Physiological differences between cold blooded and warm blooded animal cells may be an important barrier to transmission, as was concluded for infectious salmon anaemia, ${ }^{21}$ w29 w30 but there are examples of avian viruses, such as avian $\mathrm{flu}^{\text {w31 }}$ and Newcastle disease, ${ }^{\text {w32 }}$ overcoming smaller temperature barriers (avian body temperature $40^{\circ} \mathrm{C}$, human body temperature $37^{\circ} \mathrm{C}$ ).

In developing a broader public health approach in which absence of evidence is distinguished from evidence of no risk, it is worth noting that human exposure is often likely to occur first in occupational groups, ${ }^{\mathrm{w} 33}$ and serosurveys of such groups are helpful. For example, the absence of infection in workers exposed to porcine circovirus suggests that this virus is not a zoonosis. ${ }^{9}$ On the other hand, seropositivity in handlers of macaques infected with simian parvovirus ${ }^{22}$ would designate it as a level 2 zoonosis and would justify enhanced surveillance of human cases, as well as extra precautions for seronegative pregnant women who might be exposed. Development of serum archives representing occupational groups at risk would be a major and urgently needed strategic response to emerging infections. ${ }^{23}$ w34-w36

The algorithm we developed provides a discipline that ensures that the strength of evidence and the adequacy of surveillance are addressed and provides stopping rules necessary for public health decision makers. We have distinguished four levels of zoonotic

\section{Summary points}

Most serious emerging infections are of animal origin

When a new animal disease emerges virological and microbiological criteria are usually insufficient to make the risk assessment of zoonotic potential

An algorithm for early qualitative public health risk assessment has been developed that exposes the strengths and weaknesses of available evidence on risk

Assessment with this approach indicated the need for further investigation of the zoonotic potential of porcine hepatitis $\mathrm{E}$, but not for porcine circovirus and bovine norovirus at this time

Systematic enhanced surveillance of occupationally exposed groups is recommended

potential, although this is not essential to the process and the usefulness of setting levels has yet to be established. The important outputs of the algorithm are the decisions made to enhance surveillance or develop and use serological tests.

We thank the members of the ad hoc panel of experts who performed one or more of the risk assessments described above: Jim Gray, Miren Iturriza, Chong-Gee Teo, Virus Reference Department, Health Protection Agency Centre for Infections, London; M Banks, Central Veterinary Laboratory, Weybridge; J Bridger, Royal Veterinary College, Camden, London; J Gentsch, Viral Gastroenteritis Laboratory, CDC, Atlanta GA, USA; D Thomas, National Public Health Service for Wales, Cardiff; and Tim Coleman, Hereford Public Health Laboratory.

Competing interests: None declared.

1 Taylor LH, Latham SM, Woolhouse ME. Risk factors for human disease emergence. Philos Trans R Soc Lond B Biol Sci 2001;356:983-9.

2 Lanska DJ. The mad cow problem in the UK: risk perceptions, risk management, and health policy development. J Public Health Policy 1998;19:160-83.

3 Cash RA, Narasimhan V. Impediments to global surveillance of infectious diseases: consequences of open reporting in a global economy. Bull World Health Organ 2000;78:1358-67.

4 Strategy Unit, Cabinet Office. Risk: improving government's capability to handle risk and uncertainty. London: Strategy Unit, 2002 (www.strategy.gov.uk/downloads/su/risk/risk/report/report/ (www.strategy.gov.uk/do
downloads/su-risk.pdf)

5 Morgan D. The Human Animal Infections and Risk Surveillance group (poster No 33). In: Proceedings of the 2nd International Conference on the Prevention and Control of Zoonoses, from Science to Policy. 2005 Jun 15-17; In: Liverpool, UK. wwwhpazoonosesconference.org.uk/ PrevCtriZoon05_posters.pdf (accessed 14 Nov 2005).

6 Meng X-J. Zoonotic and xenozoonotic risks of the hepatitis E virus. Infect Dis Rev 2000;2:35-41.

7 Ijaz S, Arnold E, Banks M, Bendall RP, Cramp ME, Cunningham R, et al. Non-travel-associated hepatitis e in England and Wales: demographic, clinical, and molecular epidemiological characteristics. I Infect Dis clinical, and molecular epidemiological
2005; 192:1166-72. (Epub 2005 Aug 22.)

8 Chae C. A review of porcine circovirus 2-associated syndromes and disChae C. A review of porcine
eases. Vet J 2005;169:326-36.

9 Ellis JA, Wiseman BM, Allan G, Konoby C, Krakowka S, Meehan BM, et al. Analysis of seroconversion to porcine circovirus 2 among veterinarians from the United States and Canada. J Am Vet Med Assoc 2000;217:1645-6.

10 Oliver SL, Dastjerdi AM, Wong S, El-Attar L, Gallimore C, Brown DW, et al. Molecular characterization of bovine enteric caliciviruses: a distinct third genogroup of noroviruses (Norwalk-like viruses) unlikely to be of risk to humans. J Virol 2003;77:2789-98.

11 Smiley JR, Hoet AE, Traven M, Tsunemitsu H, Saif LJ. Reverse transcription-PCR assays for detection of bovine enteric caliciviruses (BEC) and analysis of the genetic relationships among BEC and human caliciviruses. J Clin Microbiol 2003;41:3089-99. 
12 Cotto E, Neau D, Cransac-Neau M, Auriacombe M, Pellegrin JL, Ragnaud $\mathrm{JM}$, et al. Borna disease virus RNA in immunocompromised patients in southwestern France J Clin Microbiol 2003:41:5577-81

13 Chalmers RM, Thomas DR, Salmon RL. Borna disease virus and the evidence for human pathogenicity: a systematic review. $Q J \mathrm{Med}$ 2005;98:255-74

14 Davidson F, Lycett C, Petrik J, Fazakerley JK. Investigation of frequency of active Borna disease virus infection in Scottish blood donors. Vox Sang 2004;86:148-50

15 Poxton IR. Clostridium difficile. J Med Microbiol 2005;54:97-100.

16 World Health Organization. Xenotransplantation: guidance on infectious disease prevention and management. In: Emerging and other communicable diseases surveillance and control. Geneva: WHO, 1998. (WHO/EMC/200/ 98.1.)

17 Boneva RS, Folks TM. Xenotransplantation and risks of zoonotic infection. Ann Med 2004;36:504-17.

18 Audelo-del-Valle J, Clement-Mellado O, Magana-Hernandez A, Flisser A, Montiel-Aguirre F, Briseno-Garcia B. Infection of cultured human and monkey cell lines with extract of penaeid shrimp infected with Taura syndrome virus. Emerg Infect Dis 2003;9:265-6.

19 Pantoja CR, Navarro SA, Naranjo J, Lightner DV, Gerba CP. Nonsusceptibility of primate cells to Taura syndrome virus. Emerg Infect Dis 2004;10:2106-12.

20 Cook N, Bridger J, Kendall K, Gomara MT, El-Attar L, Gray J. The zoonotic potential of rotavirus. J Infect 2004;48:289-302.

21 Scientific Committee on Animal Health and Animal Welfare. Assessment of zoonotic risk from infectious salmon anaemia virus. Brussels: European Commission Health and Consumer Protection Directorate-General, 2000. (http://europa.eu.int/comm/food/fs/sc/scah/out44_en.pdf).

22 Brown KE, Liu Z, Gallinella G, Wong S, Mills IP, O’Sullivan MG. Simian parvovirus infection: a potential zoonosis. J Infect Dis 2004;190:1900-7.

23 Thomas DR, Salmon RL, Coleman TJ, Morgan-Capner P, Sillis M, Caul $\mathrm{EO}$, et al. Occupational exposure to animals and risk of zoonotic illness in a cohort of farmers, farm workers and their families in England. J Agric Saf Health 1999;5:373-82.

\title{
Commentary: Zoonotic potential of emerging animal diseases
}

\author{
Samson S Y Wong, K Y Yuen
}

Research Centre of Infection and

Immunology,

Department of

Microbiology, the

University of Hong

Kong, Queen Mary

Hospital, Hong

Kong, China

Samson S Y Wong

assistant professor

K Y Yuen

professor of infectious

diseases

Correspondence to:

K Y Yuen

hkumicro@

hkucc.hku.hk
Palmer and colleagues have proposed an algorithm for early qualitative risk assessment of the emerging zoonotic potential of animal diseases, ${ }^{1}$ a vital problem since more than half of all new or emerging infectious diseases agents in humans are zoonotic in origin. ${ }^{2}$ Human infections due to agents such as the coronavirus responsible for severe acute respiratory syndrome (SARS), avian influenza A viruses, and HIV pose enormous problems because they are $(a)$ difficult to manage clinically, $(b)$ prohibitively expensive to treat in resource-poor areas, $(c)$ capable of rapid global spread, (d) virtually impossible to eliminate once stable transmission among humans has been established, or (e) capable of inducing fear and substantial economic losses. Therefore, prior knowledge and public health preparedness are essential for their prevention and control. The bottleneck for this control effort lies in discovering and characterising these agents. Once achieved, these should be followed by systematic analyses of the risk of the agents causing human diseases.

Using porcine hepatitis $\mathrm{E}$ virus, porcine circovirus, bovine norovirus, Borna disease virus, and Clostridium difficile as examples, Palmer and colleagues systematically analysed the available scientific and clinical data on the microbes and the microbe-host interactions, and gave recommendations on the level of confidence of their risk of zoonotic transmission. ${ }^{1}$ Their work exposed the fact that current knowledge is often insufficient to exclude the possibility of human infections.

In many disease syndromes where the aetiological agents cannot be defined or when the syndrome is conventionally regarded as idiopathic, clinicians often fail to explore the history of animal exposure and do not order microbiological tests for zoonotic agents. Many of these tests are not routinely available in local hospital laboratories, and serological tests for animal diseases are generally not standardised for testing human samples.

When zoonotic transmission occurs, some mutations might have occurred which could impair the sensitivity of rapid tests such as nucleic acid amplification. Most scientists agree that the mechanisms of interspecies jumping between viruses are poorly understood. It takes only a single amino acid change to alter the receptor binding specificity of the haemagglutinin of influenza A $\mathrm{H} 5 \mathrm{~N} 1$ virus, allowing the virus to be trans- mitted from chicken to human rather than just chicken to chicken. ${ }^{3}$ Similarly, the substitution of one amino acid in the surface spike protein of SARS coronavirus changes its specificity from civets to human. ${ }^{4}$

Thus, any preceding epidemiological, clinical, and microbiological analysis may not be able to foretell such events-with potentially catastrophic consequences. As a result, it would not be possible to make confident recommendations for public health decisions or risk communications to the public without continuous research effort and a comprehensive surveillance programme. Continuous serological and disease monitoring for workers with frequent animal exposure could be a sentinel system for this surveillance programme.

The search for new microbial agents in animals is equally important because the simian immunodeficiency virus and bat SARS coronavirus were discovered shortly after human infections with SARS coronavirus and HIV were noted. ${ }^{56}$ The human coronavirus OC43 is believed to have been acquired after an interspecies jump from bovine coronavirus in the 19th century. ${ }^{7}$ It is theoretically possible that such precursor or related viruses could be discovered once a family of virus is known to exist by a comprehensive virological search in animals. This should, of course, be followed by regular monitoring of its evolution and spread in animals. Coupled with the surveillance in occupationally exposed people, it might give us a better idea of the zoonotic risk of these agents.

Palmer S, Brown D, Morgan D. Early qualitative risk assessment of the emerging zoonotic potential of animal diseases. BMJ 2005;331:1256-60. Taylor LH, Latham SM, Woolhouse MF. Risk factors for human disease emergence. Philos Trans R Soc Lond B Biol Sci 2001;356:983-9.

Gambaryan A, Tuzikov A, Pazynina G, Bovin N, Balish A, Klimov A. Evolution of the receptor binding phenotype of influenza A (H5) viruses. Virology (in press).

4 Yi CE, Ba L, Zhang L, Ho DD, Chen Z. Single amino acid substitutions in the severe acute respiratory syndrome coronavirus spike glycoprotein determine viral entry and immunogenicity of a major neutralizing domain.J Virol 2005;79:11638-46

Guan Y, Zheng BJ, He YQ, Liu XL, Zhuang ZX, Cheung CL, et al. Isolation and characterization of viruses related to the SARS coronavirus from animals in southern China. Science 2003;302:276-8.

Lau SK, Woo PC, Li KS, Huang Y, Tsoi HW, Wong BH, et al. Severe acute respiratory syndrome coronavirus-like virus in Chinese horseshoe bats. Proc Natl Acad Sci USA 2005;102:14040-5.

7 Vijgen L, Keyaerts E, Moes E, Thoelen I, Wollants E, Lemey P, et al. Complete genomic sequence of human coronavirus OC43: molecular clock plete genomic sequence of human coronavirus OC43: molecular clock
analysis suggests a relatively recent zoonotic coronavirus transmission event. J Virol 2005;79:1595-604. 